\title{
An update on THORPEX-related research in data assimilation and observing strategies
}

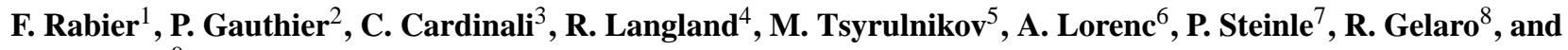 \\ K. Koizumi ${ }^{9}$ \\ ${ }^{1} \mathrm{CNRM} / \mathrm{GAME}$, Météo-France and CNRS, France \\ ${ }^{2}$ Department of Earth and Atmospheric Sciences, UQAM, Montréal, Canada \\ ${ }^{3}$ European Centre for Medium-range Weather Forecasts, Reading, UK \\ ${ }^{4}$ Naval Research Laboratory, USA \\ ${ }^{5}$ Hydrometeorological Research Centre of Russia, Russia \\ ${ }^{6}$ The Met-Office, UK \\ ${ }^{7}$ Bureau of Meteorology Research Centre, Australia \\ ${ }^{8}$ NASA/Global Modelling and Assimilation Office, USA \\ ${ }^{9}$ Japanese Meteorological Agency, Japan
}

Received: 13 August 2007 - Revised: 22 November 2007 - Accepted: 20 December 2007 - Published: 8 February 2008

\begin{abstract}
The international programme "THORPEX: a World Weather Research Programme" provides a framework in which to tackle the challenge of improving the forecast skill of high-impact weather through international collaboration between academic institutions, operational forecast centres, and users of forecast products. The objectives of the THORPEX Data Assimilation and Observation Strategy Working Group (DAOS-WG) are two-fold. The primary goal is to assess the impact of observations and various targeting methods to provide guidance for observation campaigns and for the configuration of the Global Observing System. The secondary goal is to setup an optimal framework for data assimilation, including aspects such as targeted observations, satellite data, background error covariances and quality control. The Atlantic THORPEX Regional campaign, ATReC, in 2003, has been very successful technically and has provided valuable datasets to test targeting issues. Various data impact experiments have been performed, showing a small but very slightly positive impact of targeted observations. Projects of the DAOS-WG include working on the AMMA field experiment, in the context of IPY and to prepare the future THORPEX-PARC field campaign in the Pacific by comparing sensitivity of the forecasts to observations between several groups.
\end{abstract}

Correspondence to: F. Rabier

(Florence.Rabier@meteo.fr)

\section{Introduction}

The international programme "THORPEX: a World Weather Research Programme" was established in 2003 by the Fourteenth World Meteorological Congress as a ten-year international research and development programme. It is now providing a framework in which to tackle the challenge of improving the skill of high-impact weather forecasts through international collaboration among academic institutions, operational forecast centres, and users of forecast products. Its mission Statement is "Accelerating improvements in the accuracy of high-impact 1-14 day weather forecasts for the benefit of society and the economy". Information on this programme, and in particular the science plan and the implementation plan, can be found on the World Meteorological Organisation web page (www.wmo.int). Among the core objectives, THORPEX plans to contribute to the design and demonstration of interactive forecast systems in which the uncertainty of the analysis and future forecasts is assessed. This estimation leads to the development of a flow and situation dependent assimilation system. In particular, it is envisaged to use targeted observations where and when extra information is crucially needed. Enhancements to the observation usage in "sensitive" regions of the atmospheric flow leads in turn to a reduction in forecast error. Other main goals are to perform THORPEX Observing-System Tests and Regional field Campaigns to test and evaluate experimental remote-sensing and in-situ observing systems, and when feasible, demonstrate their impact on weather forecasts. Major field experiments have already been conducted and will continue to be organised, during which many ideas can be tested and new observations can be assimilated.

Published by Copernicus Publications on behalf of the European Geosciences Union and the American Geophysical Union. 

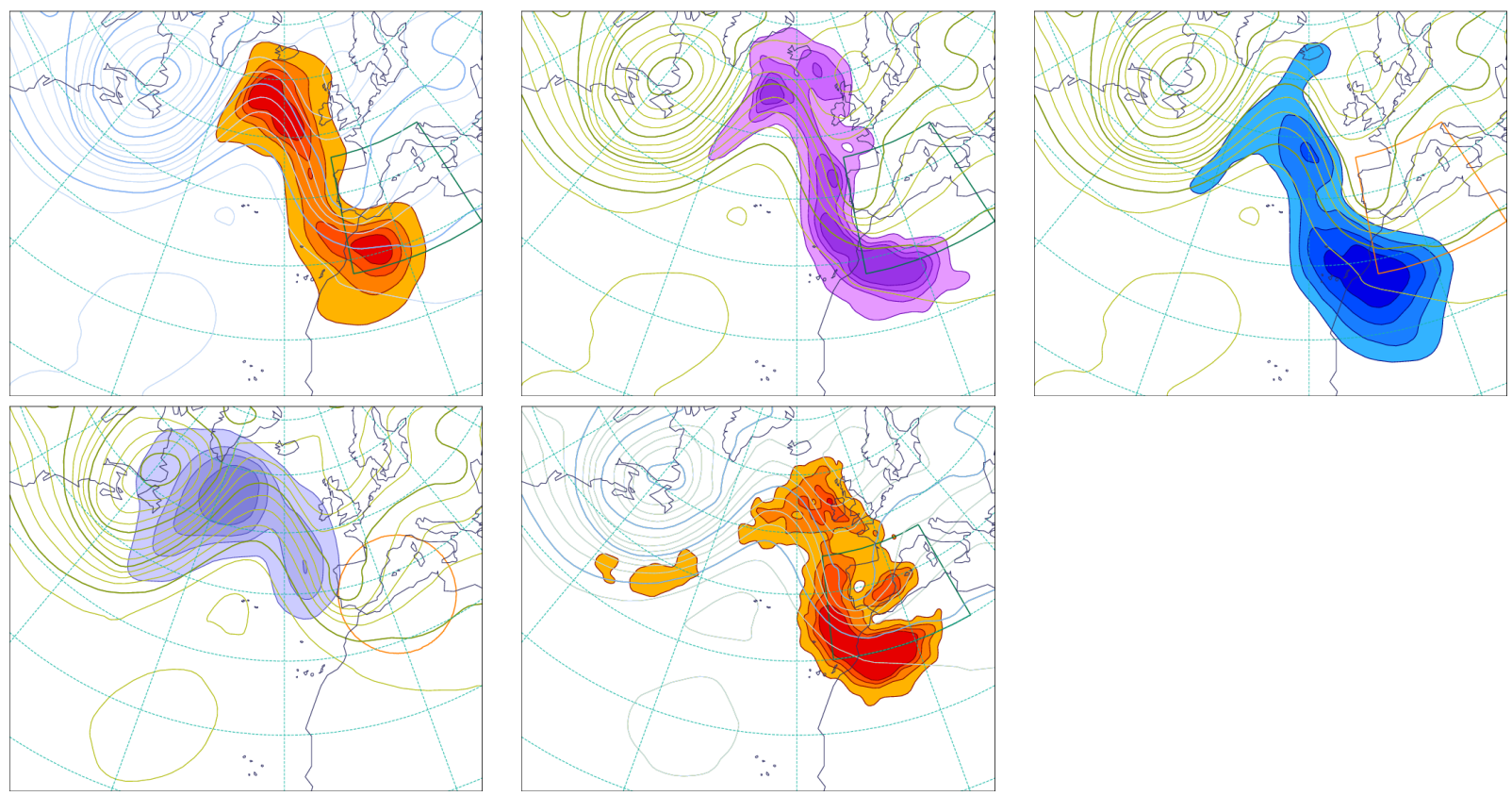

Fig. 1. Example of ATReC targeting guidance maps: ATReC case 12.3. Targeting date (valid time for the sensitive areas) is the 15 of Nov. 2003 at 18:00 UTC. The verification region, valid $42 \mathrm{~h}$ later on the 17 of Nov. at 12:00 UTC, is centred on the point $37^{\circ} \mathrm{N}-02^{\circ} \mathrm{E}$ with a $1000 \mathrm{~km}$ radius. This area is indicated in the figure. Upper left frame shows technique ecmf1 (Ecmwf total energy dry T42 singular vectors), upper frame in the middle shows ecmf2 (Ecmwf total energy moist T95 singular vectors), upper right shows ecmf3 (Ecmwf Hessian dry T42 singular vectors). The lower frames show egrr (Met Office ETKF, working with a circular verification region) on the left and lfpw (MétéoFrance total energy dry T63 singular vectors) in the middle. The shaded contours correspond to the ATReC plotting convention: they circle areas of surface 1,2,4 and 8 millions of squared kilometres, where the vertically integrated total energy of the combined singular vectors is the largest. The overlaid fields are the $500 \mathrm{hPa}$ geopotential height, valid at targeting time, i.e. they are forecasts initialized at analysis time with a lead-time range and originating either from Météo-France (panel e) or Ecmwf (all the other panels). (Courtesy : A. Doerenbecher).

The objectives of the Data Assimilation and Observation Strategy Working Group (DAOS-WG), co-chaired by P. Gauthier and F. Rabier, are two-fold. The primary goal is to assess the impact of observations and various targeting methods to provide guidance for observation campaigns and for the configuration of the Global Observing System (GOS). The secondary goal is to set-up an optimal framework for data assimilation, including aspects such as targeted observations, satellite data, background error covariances and quality control.

Tools developed in the community and data from field experiments can be used to investigate these main issues. A broad participation to this group is welcome. Collaboration between academic researchers and weather centres is encouraged.

This paper will give an overview of the objectives of the DAOS-WG in Sect. 2, will present the first results obtained by the WG in Sect. 3 and plans for future work in Sect. 4, before concluding in Sect. 5 .

\section{Main objectives}

\subsection{Observing system design}

The main goal is to develop and test systematic and objective techniques for the design of observing networks. It can be sub-divided into various tasks.

\subsubsection{Assessing the impact of observations and evaluating potential new data streams}

Operational data used at Numerical Weather Prediction (NWP) and research centres consist of various data types, provided by the GOS. The backbone of this system is formed by surface observations from land and ship stations, and vertical soundings from radiosonde and pilot balloons. Since the 1970s, other data types emerged, such as drifting buoys, aircraft measurements, wind profilers, satellite radiances, satellite cloud-drift winds and scatterometers. On one hand, observations such as land stations and radiosonde observations have been providing a stable source of information throughout the years, but their horizontal distribution is far from being homogeneous. On the other hand, satellite observations 
are expanding rapidly and becoming a major, horizontally homogeneous, source of information in current systems but are more complex to use in data assimilation. An important question arises in the use of observations: can the information provided by the observations be quantified? A simple data count might be misleading as not all observations are equal in what they measure and in their accuracy. To address this, diagnostics of the impact of observations in the data assimilation process have been developed. The main ones are briefly presented here.

To measure the impact of observations on the analysis, the Degrees of Freedom for Signal (DFS) can be used (Purser and Huang, 1993; Rodgers, 2000; Fisher, 2003; Cardinali et al., 2004; Chapnik et al., 2006). It is algebraically defined by $\mathrm{DFS}=\operatorname{Tr}(\mathrm{HK})$, where $\mathrm{H}$ is the linearised observation operator and $\mathbf{K}$ is the Kalman gain matrix. The trace of the HK matrix quantifies the gain in information brought by independent observations in the analysis. The DFS characterizes how the assimilation system uses the observations to pull the analysis away from the background. It corresponds to the partial derivative of the analysis in observation space to the observations. Although this measure of information is not necessarily translated into value in terms of forecast impact, some results about the relative importance of data actually correlate well with recent data-impact studies. Another measure of observation impact, which, contrary to the previous one, is not statistical but relative to individual cases, explicitly includes the role of the observed innovation covariance. It is equal to the covariance of the increment $x_{a}-x_{b}=\mathbf{K}(\mathrm{y}-$ $\left.\mathrm{H}\left(x_{b}\right)\right)$, where $x_{a}$ is the analysis and $x_{b}$ the background. In the optimal case, the covariance of this signal due to the observations is equal to the reduction in error variance due to the assimilation. Both these quantities can be used in observational network design strategies (Bishop et al., 2001 and 2003).

Various methods exist to quantify data impact on the forecast. A traditional way of estimating data impact in a forecasting system is to perform Observing System Experiments (OSE) such as those illustrated in Bouttier and Kelly, 2001 or Kelly, 2007. OSEs can be performed in two ways: in one way, the performance of a baseline (reference) experiment which uses a minimum amount of observation types is compared with experiments that uses at least one additional observation type (Kelly, 2007). The other way consists in removing one particular dataset, or various datasets over a long assimilation period and compare the performance with respect to the control experiment, which assimilates the available observations from the global network. This procedure can be quite costly if one wants to perform a fine analysis of the various contributions of the elements of the GOS.

Another important development has been the ability to compute the sensitivity of the forecast to the observations with the adjoint of the data assimilation process together with the adjoint of the forecast model (see Baker and Daley, 2000; Doerenbecher and Bergot, 2001; Langland and Baker, 2004;
Cardinali and Buizza, 2004; Zhu and Gelaro, 2008). This allows one further step in the computation of the sensitivity with respect to observations and can be quite valuable, for example in the context of field experiments. It is thus possible to estimate the impact of observations on a 24-h forecast, say. The results depend on the different components of the assimilation system including the model. Moreover, the computation of the sensitivities involves a metric like total energy (moist or dry), over a given area, which can also be global. All those factors have an impact on the results.

Other methods are ensemble-based (Khare and Anderson, 2006 or Tan et al., 2007) or simulate observations with OSSEs (Observing System Simulated Experiments, such as the one developed in the THORPEX context, www.emc. ncep.noaa.gov/research/osse/). One attraction of OSSEs is that they provide a framework in which it is easier to understand/diagnose the impact of observations. As such, they provide a valuable research and pedagogical tool. OSSEs have been used for several years to try to measure the impact of proposed instruments before they are launched (e.g. Atlas, 1997; Masutani et al., 2007). OSSEs require an extensive validation and calibration. Synthetic observations must first be generated from a nature run that needs to be as realistic as possible using state-of-the-art numerical models. Once synthetic observations are available, the results of OSSEs should be validated against those of real OSEs. Considering all this, it is important to realize that the calibration of such a system may be as complex as that of a real system. For example, a study using the synthetic data base would have to do bias correction of satellite radiances as those also depend on the assimilating model. Finally, the assessment of the value of new observations depends on the assimilating system, flow regimes, etc. Even then, the results need to be viewed with caution. As we have just seen, the impact of observations in the analysis and forecast can be evaluated in different ways. Certainly, it is assimilation-system-dependent and relates to flow-dependent structure functions. Studies are needed on the evaluation of observation impact with different systems. The method itself used to quantify the impact of the observations can alter our perception and it is important to compare different approaches to make firm statements on the value of a particular type of observations. In any case, the assessment of the impact of observations is expected to provide guidance for observation campaigns, for the assessment of the value of targeted observations or new observing systems and for the configuration of the Global Observing System.

\subsubsection{Evaluating and improving targeting strategies}

The concept of adaptive observing systems, also known as targeting, has been introduced in field experiments such as FASTEX (Fronts and Atlantic Storm-Track EXperiment), NORPEX (North-Pacific Experiment) at the end of the 90's to address the issue of improving individual forecasts of active weather events. Targeting will be briefly introduced in 


\section{Relative difference in RMEE SLP}

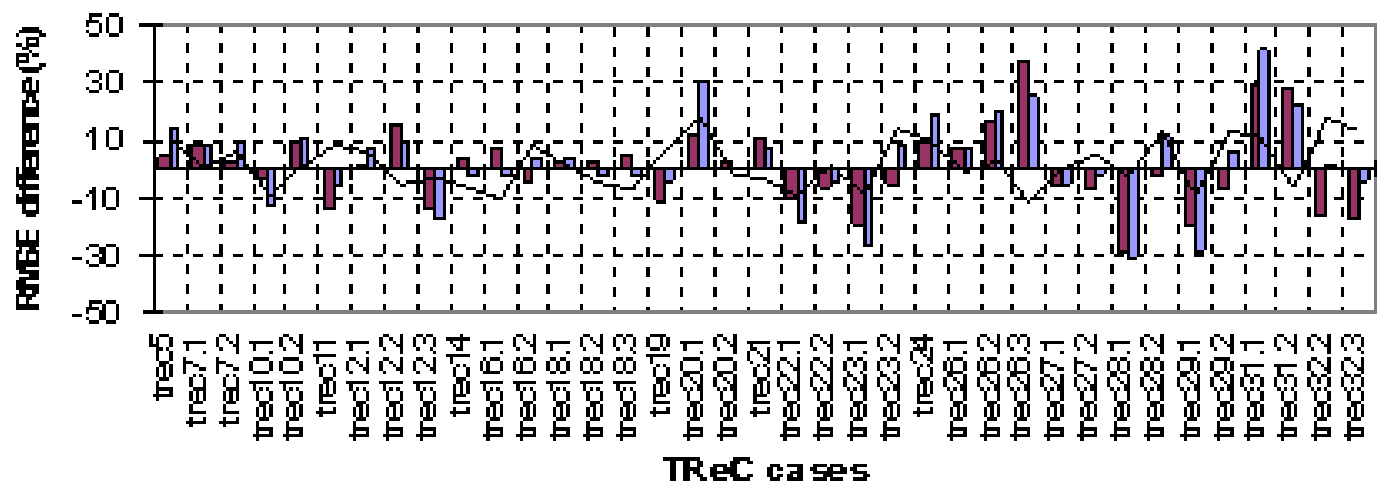

שimpr_naarg (\%) שimpr_ratrec (\%) _-Diff natrec-rat ang (\%)

Fig. 2. Impact of targeted observations during ATReC, evaluated at the Met Office. The relative improvement and deterioration in the non-cycled experiment A-targ (red) and cycled experiment ATReC (blue) of MSLP with respect to the Control forecasts for all events. Improvements are shown by an upward bar, deteriorations by a downward bar. (Courtesy of G. N. Petersen and R. Dumelow).

the following paragraph (for a more complete discussion, see the paper by Langland, 2005). In the last decade, strategies were developed to identify locations where additional observations would provide maximal improvements in the expected accuracy of forecasts. Targeting strategies are based on techniques that predict, prior to the actual measurements, the influence of an observation (or set of observations) on the uncertainty of a subsequent forecast. Different targeting techniques have been developed: some involve the adjoint of the linearized version of the forecast model and/or of the assimilation scheme, others manipulate ensembles of forecasts. This concept is currently operational in the US and is called the Winter Storm Reconnaissance Programme (WSRP, Szunyogh et al., 2000). The National Centers for Environmental Prediction use an assumption that forecast and analysis error is spanned by ensemble perturbations to solve the 4dimensional state estimation equations and predict the reduction in forecast error variance that would result from each of a set of pre-determined flight plans (Bishop et al., 2001). They also make use of simplified representations of the routine and adaptive components of the observational network (Majumdar et al., 2002). It should be noted that adjoint and Ensemble Transform Kalman Filter (ETKF) approaches are not mutually exclusive. Leutbecher's (2003) approach amounts to an application of the ETKF to an ensemble of singular vector perturbations. This fusion allows the benefits of the ETKF (quantitative predictions of reduction in forecast error variance for specific observation deployments) to be fused with those of singular vectors (subspace of the most rapidly growing perturbations relevant to the verification region).
Apart from being used for selecting additional observations, targeting observing systems can be extended to other applications like the control of the sampling rate of satellite sensors or to conduct effective thinning of the observations. This capability will become increasingly important, given the very large numbers of observations that will be available from next-generation satellites. An optimisation of the use of observations can be the selection of the most valuable subset of data, if some of the observing systems provide too many pieces of information for the data processing capabilities. One of the new challenges in data selection is the handling of advanced infrared sounders which provide thousands of radiance measurements at every observation location. The first instrument with kilo-channel data is the Atmospheric InfraRed Sounder (AIRS) on the Aqua satellite launched by the National Aeronautics and Space Administration (NASA) in 2002. On the European side, the French space agency Centre National d'Etudes Spatiales (CNES) and the European Meteorological Satellite organization (EUMETSAT) have developed the Infrared Atmospheric Sounding Interferometer (IASI), launched in 2006. For operational NWP systems, these data provide temperature and humidity information with a vertical resolution much finer than that of current instruments. The number of individual pieces of information is not usable in an operational NWP context, and several possibilities are being investigated to choose an "optimal" subset of data. This would allow extraction of the maximum information content from hyperspectral sounders, with a reduced number of individual data. An example of a channel selection procedure is presented in Rabier et al. (2002) for simulated IASI spectra (8461 radiance data). The choice of channels is based on information content with respect to 
the background or current analysis information, but it is done statically for a set of atmospheric profiles, and is not adapting to the current meteorological conditions. Among the tools which can be used for targeting, the sensitivity of the forecast to individual data can also help to select channels in an adaptive manner (Fourrié and Rabier, 2004).

What are the limitations in targeting, as it has been investigated until now? First of all, the number of cases is limited. Furthermore, targeting results are dependent on flow regimes (Cardinali et al., 2007), which limits even more the statistical significance of the results for short periods. It might also be dependent on the assimilation system used (Kelly et al., 2007). There are also some limitations due to model deficiencies (model error) and Tangent-linear/Adjoint models (e.g. physical parameterizations). Existing targeting techniques should also be generalized to account for nonlinearity and non-normality, especially in the medium-range and/or in flow regimes where physical processes like moist convection and clouds play a dominant role. The emphasis for targeting campaigns has been mostly focused on the Extra-Tropics and the extension of targeting to the Tropics still remains to be explored. This would require investigation of model deficiencies for Tropical regimes and improvements of physical parameterizations employed in the assimilation. Although it is agreed that the current diagnostics used to evaluate forecasts provide a good assessment of the validity of forecasts, they may not be sufficient to know whether these improvements are relevant for applications. Use of appropriate metrics for socio-economic applications should be defined and tested to make a more relevant assessment of forecast improvements. The evaluation of the quality of the large-scale components from global forecasts are likely to also have impact in regional forecasts at higher resolution with limited-area models which use global forecasts to drive their boundary conditions. It would be important to assess how these large-scale improvements impact the local short-term forecasts needed to determine key weather elements for socio-economic impact studies.

\subsection{Data assimilation tasks}

The goal is to optimize the data assimilation system, to make the best use of observations in an adaptive context. We will only consider existing assimilation algorithms which are based on statistical linear estimation (except the Ensemble Kalman filter, which is nonlinear as concerns dynamical evolution). These algorithms can be fully optimal, in the sense of Bayesian estimation, only under the conditions of linearity and gaussianity. It is perfectly conceivable that, just as major changes in assimilation algorithms have occurred in the past, new systems will emerge in the future.

\subsubsection{Improved use of observations}

Estimation of observation-error statistics is necessary for efficient data assimilation. The only way to acquire observation-error characteristics is to compare them (in one form or another) with other observations or a numerical forecast (see Desroziers et al., 2005 for a recent development in this field). The main problem is that, in general, it is very difficult to distinguish between the errors of the observation type in question, on one hand, and the observations/forecasts selected for comparison, on the other. Therefore, by performing specific observation campaigns, the observations in question can be compared with high-quality observations, whose error structure is known. An important point to mention is that different observation types not only have different error structures but also differ in what they measure (e.g. substantial horizontal averaging in most remote observations as compared to in situ observations). Another significant error component, which should be accounted for in data assimilation and whose error structure is to be estimated, is the representativeness error associated with forward-models. Developments specifiying the representativeness error characteristics such as those described in Frehlich (2006) are needed. Observation error includes biases, which are usually estimated and removed through bias correction schemes that have limitations. The estimation of these biases is an element of crucial importance. Work on more advanced bias correction techniques such as variational bias correction (Dee, 2005; Auligné et al., 2007) has to be encouraged.

The presence of observation error correlation is also a seriously limiting element that reduces considerably the volume of assimilated data. For instance, Bormann et al. (2003) have shown a significant horizontal correlation for atmospheric motion vectors. Idealised studies by Liu and Rabier (2003) have shown that, when assimilating observations with correlated errors in a scheme like most operational systems which is sub-optimal in not accounting for the correlations, increasing the observation density beyond a threshold can be harmful. Usually, there is a horizontal thinning of satellite data performed in a rather ad hoc manner. More advanced methods are under consideration (Ochotta, 2005). More general solutions could be preferable. In particular, instead of performing a thinning of the observations, one might prefer to perform an averaging of neighboring observations as this is performed for instance for geostationary clear-sky radiances provided to NWP users. The advantage of this type of "super-obbing" is that more information is kept in the process, which translates into smaller errors in the resulting "super-obs" compared to an individual observation. The best theoretical framework might well be to model the correlations in the long term.

Nonlinearity in the observation operator is another issue that has to be dealt with. In particular, some observations, such as satellite radiances and scatterometer data, are indirect measurements of atmospheric variables. For radiances, 


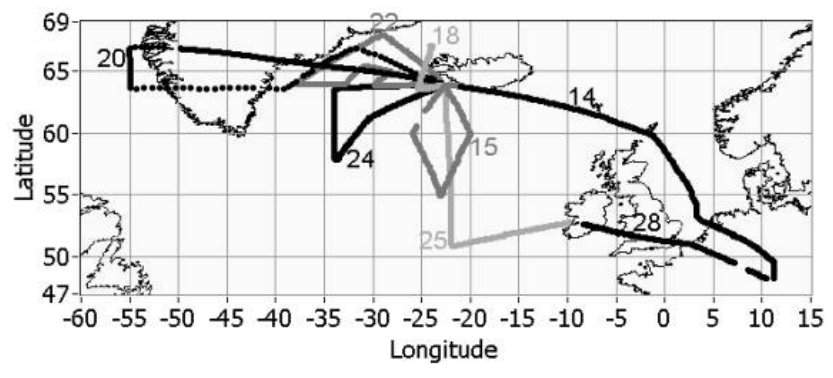

Fig. 3. Flight tracks of the Wind Lidar flights on board aircraft during the ATReC. Numbers indicate the date of the flights in November 2003. (from Weissman and Cardinali, 2007).

nonlinearity is particularly severe in the case of cloudy/rainy conditions. For scatterometer data, the observed signal is a back-scattered cross-section, which depends very nonlinearly on the surface wind over the ocean. This leads to ambiguous wind solutions, which the analysis has to handle. Possible non-Gaussianity of satellite observation errors should also ideally be allowed for. Quality control procedures also need to be improved. Interesting developments exist in the context of quality control, such as flow-dependent tolerances for outlier observations for an adaptive "buddy check" (Dee et al., 2001).

Given that only a small fraction of all incoming data makes its way to the assimilation, it is important to improve our ability to better use them. This requires that progress be made on the assimilation of cloudy and rainy radiances, which involves serious nonlinearities (infrared and microwave, e.g. Bauer et al., 2006) and on the assimilation of low-peaking channels over land with a better representation of emissivity (e.g. Karbou et al., 2006).

\subsubsection{Adaptive data assimilation}

Most operational centres have adopted 4-D-Var but ensemble approaches are also considered by many (Hamill and Snyder, 2002; Lorenc, 2003; Houtekamer and Mitchell, 2005; Hunt et al., 2007). Promising results have been obtained with quasi-operational systems which may lead to breakthroughs in data assimilation methods, particularly in the context when model error is present. Model error should also be inserted in a weak-constraint 4-D-Var (Fisher et al., 2005). The combination of variational methods and ensemble approaches to provide the analysis with flow-dependent structure functions is also promising (Etherton and Bishop, 2004; Buehner, 2005) and has to continue to be evaluated.

With the objective of improving forecasts of high impact weather, the onset of instability is often associated with dynamical elements that cannot easily be dealt with. It is important to study the way an assimilation scheme corrects the dynamical fields and to find ways to improve the assimilation of information related to those dynamical processes.
Key information of the tropopause height and temperature is needed. However, the tropopause height is altered by the presence of warm temperature in thin layers that can propagate very quickly (e.g., in a day) to the tropopause, as seen in singular vectors. Currently, the assimilation is not able to properly resolve such thin structures, and innovative work is needed. There is also a need to analyse correctly the waveguide position as it can crucially impact the subsequent highimpact weather (Davies, 2006).

When it comes to high impact weather, mesoscale high resolution models are needed to adequately represent the weather elements that are of importance. However, it is generally acknowledged that it is not sufficient to simply downscale the large-scale forecasts and several issues must be addressed to progress on improving precipitation forecasts and other high impact local weather elements (e.g., surface winds, precipitation, visibility). Forecasting with limitedarea models has special needs. The multi-scale nature of the problem means that large-scale components are needed to represent the evolution of smaller-scale features. However, those large-scale components are not well represented by a Limited Area Model (LAM) and the results depend critically on the adopted nesting strategy to propagate information from the driving model. Specific data assimilation issues are raised at the small scales. For example, several situations depend on accurate surface analyses for temperature, soil moisture, vegetation and soil characteristics. Current methods are not well suited to produce cloud analyses. At the moment, information about the height of the cloud top cannot be used adequately. Similarly, it is important to have a good representation of the boundary-layer. A vertical representation of humidity is important not only for cases with precipitation but also in dry conditions: this plays a key role in the progress of wild fires. At small scales, there are few relationships to constrain the assimilation by imposing appropriate dynamical balances. There are also significant nonlinear processes which are often associated with non-Gaussian error probability distributions, for humidity forecast errors in particular. These have to be handled adequately in the analysis system. Strong nonlinearity of most physical processes also implies that special care must be taken when using data assimilation methods based on linearization assumptions such as 4$\mathrm{D}$-Var for instance. Finally, model error is an important issue when it comes to forecasting the evolution of precipitation and clouds.

The strong constraint 4-D-Var puts limitations on the length of the assimilation window. It is now believed that a weak-constraint 4-D-Var could extend the assimilation window over longer periods. In ensemble forecasting and data assimilation, several ad hoc approaches to account for model errors are common (stochastic physics, varied model, multimodel), but in 4-D-Var, we have to explicitly specify modelerror covariances. To do this in a consistent way, a modelerror stochastic model is to be introduced. Results of a simulation study (Tsyrulnikov 2005) indicate that such a model 
should account for, firstly, the state-dependent part of model errors, secondly, for temporal correlations, and thirdly, for the fact that model errors are largely advected with the velocity that resembles Rossby-wave phase velocity for barotropic dynamics. In a model-error model, model errors are flowdependent in two respects: first, the state-dependent part of model errors is flow-dependent by definition, and second, all the parameters of the model can and should be made dependent on the local flow structure. Additional research to extend the results of Tsyrulnikov (2005) to physical (not just dynamical) errors (convection, clouds, precipitation etc.) is needed. With high-resolution forecast models and Kalman-filter or weak-constraint 4-D-Var based data assimilation, an adequate model-error model is indispensable. Model error estimation techniques are to be devised. It is important to investigate the capability of forecast ensembles to serve as a tool for model-error model identification and estimation. The THORPEX Interactive Global Grand Ensemble (TIGGE, tigge.ecmwf.int) in particular might provide an ideal framework to perform such studies. Independent observation-based techniques are also needed to complement the ensemble-based studies.

\section{First results in the context of the ATReC campaign in 2003}

The objective of the ATReC (Atlantic THORPEX Regional Campaign) was to test the hypothesis that short term forecast errors over Europe and the Eastern seaboard of the USA can be reduced by targeting extra observations over sensitive areas determined each day by the forecast flow patterns using NWP techniques (Mansfield et al., 2004). The field campaign took place in the autumn of 2003. It was an attempt at real-time adaptive control of a full set of operational observing systems (in an international context) in addition to the deployment of research aircraft. It was the first experiment to involve so many types of instruments, including commercial aircraft, in the targeting. The observations available for targeting were:

\section{Sixty-six European and Canadian radiosonde stations}

2. The EUCOS (European Composite Observing System) ASAP Fleet (13 ships)

\section{The EUCOS AMDAR Fleet (550 aircraft)}

4. Dropsondes from the NOAA G-IV, the University of North Dakota 'Citation' aircraft, the DLR Falcon and a USA Air Force C130 aircraft.

\section{Super-rapid-scan winds from the GOES satellite}

A virtual Operations Centre was set up at the UnitedKingdom Meteorological Office ("The Met Office") HQ in

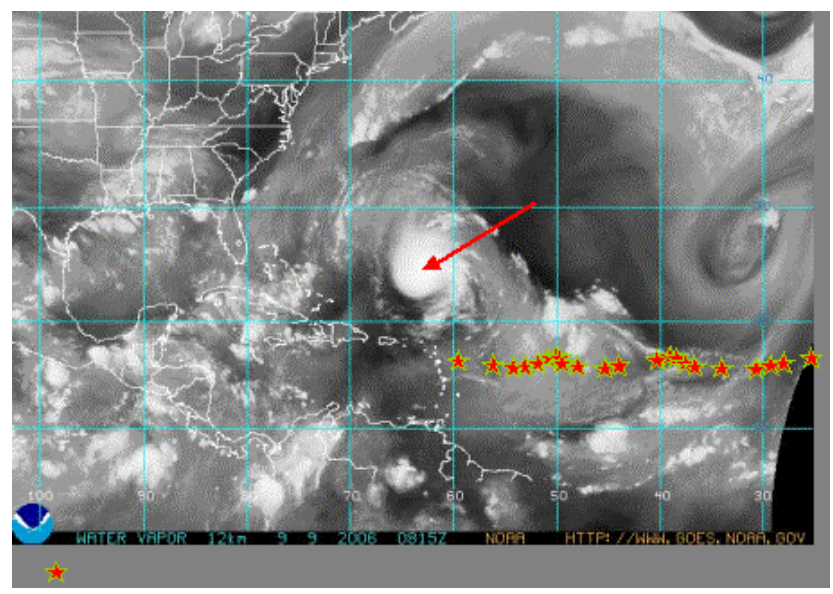

Fig. 4. Satellite image of tropical cyclone Florence (location indicated by the red arrow), together with locations of soundings from driftsondes (indicated by red stars). The satellite image is the water vapour channel from the GOES satellite on 9 September 2006 at 8:15 Z. The 23 dropsondes were released along the trajectory of the third driftsonde flight, between 5 September 2006 at 12:00 TU (latitude $25^{\circ} \mathrm{W}$ ) and 9 September 2006 at 12:00 TU. (Courtesy of D. Parsons, P. Drobinski, P. Cocquerez).

Exeter to manage the field campaign. Each morning forecasters from ECMWF, Météo-France and the Met Office separately studied NWP output for potential cases of high impact, high uncertainty or both within 3-5 day forecasts for the relevant areas. Although the aim was to improve 1-3 day forecasts it was necessary to look this far ahead in order to give $48 \mathrm{~h}$ warning to observation providers.

Different model diagnostics were available in the different centres and it was useful to compare techniques. Two diagnostics were particularly useful - a measure of the ensemble spread and the probability of weather events with thresholds and time periods set by the forecaster (calculated by ECMWF). In practice it proved fairly easy to agree on potential high impact weather and uncertainty - cases of the former were few during the period.

Case selection included defining the verification area, date and time and the observation date and time when additional data would be provided. The latter was normally centred on 18 UTC to give daytime flights for aircraft based on the western side of the Atlantic and because there are few radiosondes at this time.

Once a case was chosen, sensitive areas were computed independently as follows:

1. ECMWF - Singular Vectors (SVs),

2. NCEP and Met Office - Ensemble Transform Kalman Filter (ETKF),

3. MeteoFrance - Singular Vectors, and

4. NRL - Adjoint Sensitivity and Singular Vectors. 


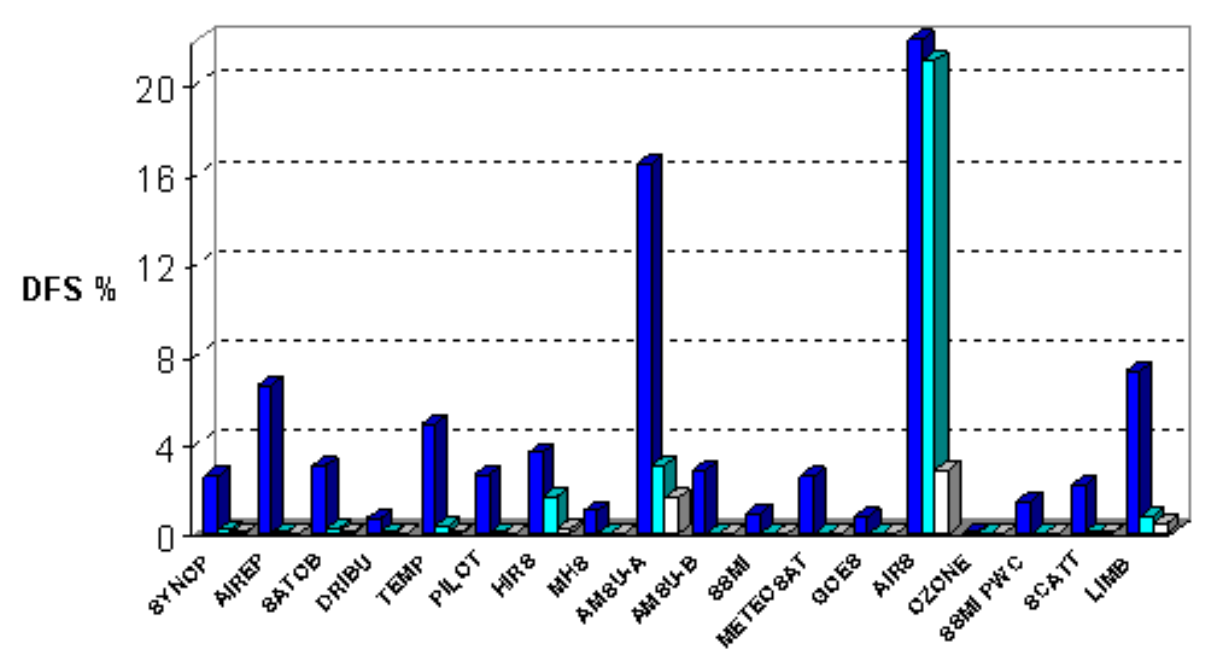

Fig. 5. Information content, i.e. degrees of freedom for signal (DFS), for the main data types in the assimilation at the ECMWF centre, in percentage. Three regions are represented: the globe (blue bars), the North Pole, north of 65N (cyan bars) and the South Pole, south of 65S (white bars). Note that the DFS for AIRS globally is $40 \%$ (truncated in the picture). The sum of all percentages for the globe is equal to $100 \%$, representing the whole contribution from observations. (Courtesy of C. Cardinali)

The resulting predictions of sensitive areas were discussed by forecasters, NWP experts and aircraft scientists at a 16:00 UTC daily conference. This was generally a more difficult process than case selection because of the need to consider the availability of the observations systems and the different estimates of the sensitive areas. In general, datatargeting areas included the areas of maximum sensitivity. Observation providers were contacted by email requesting the additional observations with a $48 \mathrm{~h}$ warning followed by confirmation the next day. The great majority of the data were available in real time on the GTS and used in the operational runs of the models.

The campaign generated 32 considered cases of which 21 triggered observations (some on multiple days). All the extra data collected amounted to some 214 additional radiosonde ascents, 277 aircraft dropsonde profiles, 65 extra ascents from ASAP ships and 23000 additional AMDAR observations. Cases ranged from European wind storms to heavy Mediterranean rainfall and US East coast rainfall/snowfall events. The data is available to all user communities. Mansfield et al. (2004) provide more details about the websites where the data can be found.

Of course the goal of targeting is to improve the forecast over a specific region at a particular time. The coherence of the sensitive area predictions computed with the various methods is important. Doerenbecher et al. (2004) have looked at this for the ATReC cases and found it was difficult to get a good consensus for flight planning purposes. As an example, Fig. 1 shows the correspondence between various targeting techniques (Doerenbecher, pers. com.). It shows examples of targeting guidance maps for dry SVs with total energy metric (ecmf1), moist SVs with total energy metric (ecmf2), dry SVs with Hessian metric (ecmf3), ETKF run at the Met Office (egrro) and dry SVs with total energy metric run at Météo-France (lfpw1). One can notice a good agreement between the various SV techniques with total energy metric, a relatively good agreement between SV techniques using total energy and Hessian metric, and a poor agreement of ETKF with SV based techniques. This is consistent with other studies (Majumdar et al., 2002 and 2006). This needs to be investigated further in the context of a systematic comparison of the effectiveness of the regions identified by differing strategies. Associated with this, sampling strategies of sensitive areas need to be studied. During this campaign and previous ones, due to practical constraints for the deployment of observations, the sampling of the sensitive areas was not ideal (see also Cardinali and Buizza, 2003 for the NORPEX 98 campaign).

Several groups have carried out a series of experiments to identify whether any improvement in the forecasts can be detected due to the addition of the targeted data. Most centres have been running two experiments, one ATReC experiment assimilating all data including extra targeted data, and a Control experiment excluding the targeted observations. These experiments are usually carried out in cycled mode, i.e. each data assimilation is run in a continuous stream. This implies that the ATReC experiment has been using targeted data continuously with indirect effects of targeted data on subsequent analyses at later times.

At Météo-France, the conclusions of Fourrié et al. (2006) are based on the analysis of the last three weeks of the campaign during which there were more targeting flights and the meteorological situations were more interesting. The forecast model was ARPEGE and the assimilation scheme was 


\begin{tabular}{lll}
\hline Case Improved & Case Worsen & Case Neutral \\
\hline $24 \%$ & $10 \%$ & $66 \%$ \\
\hline
\end{tabular}

the operational 6-h 4-D-Var of Météo-France. The quality of forecasts has been assessed with respect to both analyses and radiosondes for many levels and parameters with much the same results. For large verification regions, the inclusion of the campaign data improves the short range $(6-18 \mathrm{~h})$ forecast very slightly, is neutral for the $24-72 \mathrm{~h}$ forecast and improves the longer range (beyond $72 \mathrm{~h}$ ). The results are found to vary with verification area. A significant improvement over central Europe was found at $100 \mathrm{hPa}$. This improvement is mainly due to the inclusion of extra radiosonde observations in the analysis. Considering the verification areas associated with each of the 22 ATReC cases (sample of the total of 32 cases, due to the shorter period) studied gives more mixed results. For example, at the $1000 \mathrm{hPa}$ level, 9 cases show improvement (by more than 10\%), 8 are neutral and 5 show degradation (by more than $10 \%$ ). At $500 \mathrm{hPa}$, the number of neutral cases increases to 14 , whilst 3 are improved and 5 degraded. On the whole, there is a small positive impact at the optimisation time $(48 \mathrm{~h})$ in the verification area. It proved difficult to establish a relation between the forecast score and the number of supplementary data in the targeted area. The largest impact obtained (case 25) had no observations in the sensitive area! This obviously comes from the cycled mode of the experiments which renders straightforward interpretation of data impact more complex. Thus the conclusion is reached that the larger the verification area the better the results (more consistent forecast improvement) - which agrees with the Desroziers et al. (2003) FASTEX reanalysis.

At ECMWF (C. Cardinali, personal communication), the impact of targeted ATReC observations has been examined by performing an OSE that consists mainly of two experiments, with and without ATReC data. Forecast impact is calculated in terms of RMS forecast errors differences between Control and ATReC normalized with respect to the RMS forecast error of the Control computed in the verification regions indicated by the targeted cases. Verification regions and forecast time vary with the case. Results are similar for the various parameters (wind components $u$ and $v$, temperature and geopotential) but they can vary with level. The results for the mean sea level pressure (MSLP) field shown in the table above are for the full observation campaign (17 Oct-15 Dec 2003): neutral means an impact between $\pm 10 \%$.

Another experiment has also been run in non-cycled mode, which corresponds to a pure targeting experiment, named A-Targ. This means that, based on the control, additional targeted data have been added to the analysis separately for each targeting case and the impact for that particular day is computed. There is no one-to-one correspondence between the improvements/degradations brought by the experiments performed in cycling and non-cycling mode. In general, the impact is smaller in the pure targeting mode, i.e. without performing a continuous cycling experiment.

To understand the relatively modest average impact of targeted data in this field campaign, further experiments were performed at ECMWF (Kelly et al., 2007; Buizza et al., 2007; Cardinali et al., 2007). Over two seasons, the value of observations in target areas over the Atlantic and the Pacific oceans was investigated by removing observations in specific areas. These areas were either chosen at random or were determined by SV calculations. In particular, Buizza et al. (2007) results show that removing all current observations in SV areas, representing around $15 \%$ of the surface of the North Atlantic ocean, increased the geopotential (250, $500,1000 \mathrm{hPa}$ ) RMS error at day 2 over Europe by about $3 \%$ in winter and $3.6 \%$ in summer, that is a factor 1.1 and 2.2 larger than removing observation in randomly located areas (same size of SV areas) in winter and summer, respectively. For the North Pacific, they concluded that observation taken in SV-target areas are more valuable than observations taken in random areas, by a factor 7.2 in winter and a factor 3.5 in summer when the verification area is North America. This is to be compared with a degradation of $45 \%$ which would be caused by removing all observations in the North Pacific and Atlantic oceans over North America and Europe, respectively. The conclusion is that, indeed sensitive areas based on SVs of the day are more effective at optimizing data impact than sensitive areas based on averaged SVs or random areas, but that the impact of removing observations over a small portion of the oceans only amounts for a small degradation of the forecast performance. This might help to explain why adding a small amount of observations in the North Atlantic is in general not very effective. However, Cardinali et al. (2007) showed that during tropical cyclone activity in North Atlantic and particularly tropical cyclone transition to extratropical characteristics, removing observations in sensitive regions degraded the forecast skill by up to $13 \%$ which is 6 times larger than when removing observations in randomly designed areas.

Observing System Experiments using global Met Office model (in 4-D-Var) have been run by the Met Office and the University of Reading for the whole of the ATReC period (Petersen and Thorpe, 2007). The experiments have compared the impact of forecasts run from analyses that used all the targeted data (ATReC) with those that did not use any targeted data (Control). The results shown in the table below indicate that the impact of the targeted data on forecasts verified over standard areas (e.g. the whole of Europe) is approximately neutral, although very slightly positive. The RMS forecast error differences in the case dependent verification area are similar as the ones from ECMWF.

Another experiment was run in non-cycled mode (A-Targ). Each of the ATReC cases is shown in Fig. 2. There is an improvement larger than 5\% for sea level pressure for the ATarg experiment in $46 \%$ of the cases. Similarly to the results 


\begin{tabular}{lll}
\hline Case Improved & Case Worsen & Case Neutral \\
\hline $24 \%$ & $16 \%$ & $60 \%$ \\
\hline
\end{tabular}

obtained at ECMWF, the cycling process seems to enhance the data impact, in both directions. However, it is not systematic, either positive or negative. On average, the lowest RMS errors are found for the A-Targ experiment.

Results from the US (Song, Toth and Majumdar, personal communication) show small positive impacts from the dropsondes, in an experiment run in cycled mode. The experiment includes 8 flights with dropsondes. Results are verified over 10 verification regions. Significantly more cases are improved than degraded. Overall results are similar to that of 2004 Winter Storm Reconnaissance Campaign (WSR04) except that humidity and wind vector wind improvements are not as significant.

At NRL, Rolf Langland performed some sensitivity to observations experiments over the period (Langland, 2005). An adjoint-based procedure is used to estimate the impact of each observation at 18:00 UTC on the 42-h forecast error. It is found that the largest impact is provided by commercial aircraft data and that targeted dropsondes have high impact per observation but low total impact because of the small amount of data.

New observing system tests were also performed during the ATReC. In particular, the airborne Doppler lidar of the Deutsches Zentrum für Luft- und Raumfarht (DLR) was used to observe wind in predicted sensitive regions (see Fig. 3 for flight tracks). In eight flights, the system measured a total of 1600 wind profiles that were experimentally assimilated at ECMWF. Results from Weissmann and Cardinali (2007) show a promising forecast impact from these additional wind information, which is encouraging for the future ADM-AEOLUS mission.

As a conclusion, the ATReC field campaign has been very successful technically and has provided valuable datasets to test targeting issues. Various data impact experiments have been performed, showing a small but very slightly positive impact of targeted observations. The impact is not always systematically positive on the verification area because of the statistical assumptions involved in the data assimilation procedure, and is not always consistent between centres. In general however, there are about twice as many cases when the forecast is improved as when it is degraded. Additional data have been found to have more impact during the last part of the field campaign (November/December) when the weather has been more unsettled. However, the overall forecast uncertainty during the campaign was small, thus a large RMSE reduction due to a small number of additional observations could not really be expected. Furthermore, the observing system resources in ATReC were not able to fully sample the identified target regions. The degree of success in sampling the target areas varied greatly from case to case, from very poor observation coverage to partial coverage of target areas. A general finding (by the two centres who performed the experiments) is that the cycling process in the data assimilation procedure enhances the results, compared to a pure targeting mode in which the data impact is computed independently on a case by case basis. Dropsondes are found to have high impact per observation, but low total impact due to the small number of additional observations. It is found that the various targeting techniques gave different sensitive areas. In particular the ETKF and SV results are noticeably different. Data denial experiments confirmed that the SV approach was relevant to find sensitive areas, but that removing data over a small portion of, for instance, the Atlantic Ocean only amounted on average to a $3.6 \%$ degradation of the forecast performance at the day-2 range over Europe in summer. This might give us an indirect confirmation that the relatively disappointing results obtained during this campaign are actually understandable based on the predictability of the flow and on the ability of data assimilation systems to cope well with a slightly reduced number of observations. However, it should be kept in mind that the forecast impact is a function of the quality of current observation coverage in the target region, the assimilation system used and the weather regimes occurring during targeted campaigns. The data denial study, here summarized, is based on the assumption that there are adequate current observations in the SV target region, which, by being located over the oceans, consists mainly of satellite data. Satellite observations can be a great potential and inexpensive target source but the limitation to observe sensitive cloud-covered regions must be further investigated. It is anticipated that adding satellite rapid-scan winds or lidar observations might be beneficial. There certainly is a potential for adding much larger amounts of observations on-demand, which could produce significant forecast impacts.

As a final remark, the data assimilation systems used operationally might not yet be able to make optimal use of data in sensitive regions where the characteristics of the error statistics can deviate significantly from the averaged values used in operational systems. More information content could probably be extracted from extra observations in a fully flowdependent system. In addition, the forecasting model might also play a role, or not, in the propagation of the signal introduced by the assimilation process.

\section{Future plans}

\subsection{AMMA and THORPEX}

The African Monsoon Multidisciplinary Analyses, AMMA (www.amma-international.org) is an international project aiming at improving our understanding of the African monsoon on timescales of one day to inter-annual. A core research goal of THORPEX is to increase knowledge of the global-to-regional influences on the initiation, evolution 
(a)

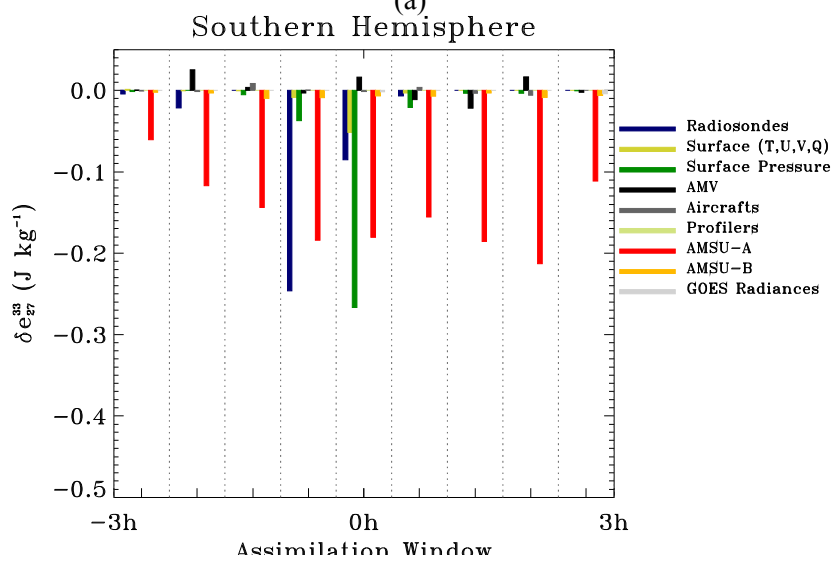

(b)

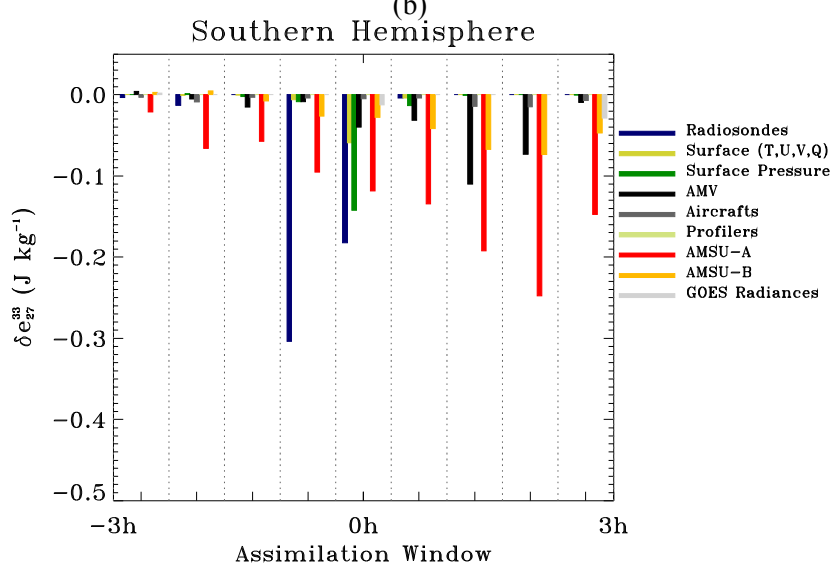

Fig. 6. Adjoint-based sensitivity to observations over the Southern Hemisphere in the Canadian system for (a) 3-D-Var, (b) 4-D-Var. The computation corresponds to the sensitivity of the total energy of the 24-h forecast error with respect to the observations. Negative values indicate that the observations contribute to an improvement in the forecast. (Courtesy of S. Pellerin, Environment Canada)

and predictability of high impact weather. A collaboration that extends measurements and modelling efforts from over Africa to across the Atlantic will allow for greater understanding of initiation, evolution and predictability of tropical convection, hurricanes and so-called ET (extratropical transition) storm systems and of tropical impacts on mid-latitude predictability. Advances in our knowledge of factors that limit the predictability of these systems will also result from these efforts. In this arena, THORPEX and the downstream AMMA goals overlap.

A joint AMMA-THORPEX working group (www.eol. ucar.edu/projects/amma-us/wg/wg5/) has been created with objectives to study sensitivities in Tropical regions and to perform a demonstration of using driftsondes. An example of driftsonde track and dropsonde deployment close to Tropical Cyclone Florence is provided in Fig. 4 (Drobinski et al., 2006). Other projects include optimizing satellite data usage over the region and finding an optimal radiosonde network.

\subsection{THORPEX-IPY}

A significant effort is taking place during the International Polar Year. A number of observation campaigns will take place in the next few years under the umbrella of the THORPEX program. Joint activities with THORPEX in data assimilation are organized in a THORPEX-IPY cluster, managed by T. E. Nordeng from Norway. It focuses on the assimilation of satellite data over polar regions. The emphasis is on polar-global interactions. Cases have been documented in which Tropical systems go through the extra-Tropical transition process to end up creating high impact weather over regions like Alaska. The IPY coincides with the launch of the operational MetOp satellite carrying an Infrared Atmospheric Sounding Interferometer (IASI), a high resolution interferometer providing measurements over thousands of wavelengths. The observations deployed during IPY in the Arctic and the Antarctic will be used to validate and assimilate IASI data and other existing infrared radiances (e.g., AIRS). This is for instance the case of the Concordiasi project (www.cnrm.meteo.fr/concordiasi/). The use of satellite infrared/microwave radiances will benefit from ice analyses to better represent surface emissivity and from better parameterizations of emissivity.

Figure 5 shows the information content, i.e. DFS, for the main data types in the assimilation for one particular assimilation cycle of the ECMWF system as an example. It is an updated version (dated 2007) of Fig. 5 from Cardinali et al. (2004). The DFS is presented for three areas: globally, and over the two polar areas. The conventional observations consist of surface observations (SYNOP from surface stations and DRIBU from buoys) and altitude data. The latter come either from AIRcraft REPort (AIREP), wind observations (PILOT) or radiosondes (TEMP). In addition, satellite observations are atmospheric motion vectors from geostationary satellites (SATOB), radiances from polar-orbiting satellite radiances (AMSU-A, AMSU-B, MHS, AIRS, HIRS and SSM/I) and from geostationary satellites (GOES and METEOSAT), surface winds from scatterometer (QuikSCAT), precipitable water content (PWC) from SSMI in cloudy/rainy areas, ozone observations (Ozone) and radio-occultation measurements (LIMB) from GPS receivers. One can see that AMSU-A and AIRS (advanced microwave and infra-red sounders respectively) are the most informative data types globally (a large part of the information comes from stratospheric levels). Among the conventional observations, radiosondes are the most informative. Over the polar areas, the analysis is even more controlled by satellite observations than globally, which justifies the effort put on satellite data assimilation in these areas. 


\subsection{An intercomparison experiment for THORPEX-PARC}

The Pacific Asian Regional Campaign 2008 (T-PARC) is a major international THORPEX field campaign developed as an outcome of the North American and Asian THORPEX planning process. T-PARC will take place between June and December of 2008 and will focus on advancing knowledge, improving prediction and society's response to i) western $\mathrm{Pa}$ cific and Asian typhoons from genesis to extratropical transition/decay, and ii) downstream high-impact weather events over North America, the Arctic and other locations whose dynamical roots and/or forecast errors are driven by aspects of the lifecycle of typhoons and other intense cyclogenesis events over east Asian and the western Pacific.

It is suggested that a formal test could be performed for inter-comparison of sensitivities using different methods (adjoint and ensemble based) for some recent periods. An agreement should be reached on the verification region(s), length of forecasts to be considered and other details. It is intended to be more of a diagnostic study, rather than a targeting study, because the goal would be to identify regions where observation coverage would be supplemented for an extended period of time (not just chasing targets of the day), such as might occur in T-PARC. Figure 6 presents an example of the sort of information that can be obtained with adjoint sensitivity of the forecast to observations. These results represent the total impact of observations on the 24-h forecast measured over a month (August 2004) in the 3-D-Var and 4-D-Var assimilation systems of Environment Canada. The region represented here is the Southern Hemisphere. The impact is represented as a function of time over the 6-h assimilation window. In 3-D-Var, the impact of surface pressure (green), radiosonde (blue) and AMSU-a (red) clearly stand out in Fig. 6a, the impact of the latter being independent of the time of the observations. The impact was measured similarly within the 4D-Var assimilation. All other components (e.g., model, observations, background and observation error statistics) are exactly the same. The striking feature of Fig. $6 \mathrm{~b}$ is that the impact of observations is more important for observations further down in the assimilation window. This shows that the impact of observations depends on the assimilation method employed and that 4-D-Var is more sensitive to the cut-off time than 3-D-Var.

The results of the proposed Forecast Sensitivity Intercomparison would be provided by the participating operational centres and research groups. The objective is to identify regions where supplemental observations and improved use of existing satellite / in-situ observations are most needed on a regular basis to improve forecast skill. The objective is mainly to extract synthetic results able to lead to practical recommendations for the evolution of the GOS.

\section{Concluding remarks}

As described in this paper, there are a number of approaches that have recently been proposed to evaluate quantitatively the impact of observations on the quality of forecasts. These sensitivities with respect to observations complement the $\mathrm{Ob}$ serving System Experiments that evaluate the impact of specific sets of observations. OSEs are routinely used by operational centres to evaluate the quality of their analysis and forecast systems. Intercomparison experiments based on OSEs are carried out to confirm the value of observing systems in different systems. The new approaches offer possibilities to get more detailed information on the impact of observations and this is actively being examined by several operational centres as a promising avenue for operational use. The impact of added observations needs to be investigated not only with respect to the forecasts but also with respect to the forecast of their variability as measured from ensemble prediction systems.

Results of the ATReC campaign in 2003 are certainly proof that a lot of knowledge could be gained by having several centres look at a given period with similar datasets and focus on specific scientific issues. From these results, it became clear that it is not appropriate to design campaigns in the Atlantic based solely on having in-situ targeted observations. Although the value per observation is greater, the overall impact on the forecasts is marginal and certainly does not justify the cost of deploying targeted observations on demand. Moreover, the operational management and coordination needed is often causing the observations to be deployed not quite in the way they should have been. However, adding satellite rapid-scan winds or lidar observations might be beneficial. There is a potential there for adding much larger amounts of observations on-demand, which could produce significant forecast impacts.

It would be important to estimate observation error statistics and correlation in particular. To achieve this, some thought must be given to the design of a relevant observation campaign. There may be Calibration/Validation $(\mathrm{Cal} / \mathrm{Val})$ campaigns being planned and it would be important to know about them and to link with them. It is important to make it known what information is needed by the assimilation about the observation error to get more information out of satellite measurements. It should be noted that nonlinear processes are often associated with non-Gaussian error probability distributions.

Finally, the objectives of observation campaigns could be focused on improving the models and parameterizations. Diagnostics based on observation departures are absolutely fundamental to monitor the observations and the model. There is value in considering the assimilation of the additional measurements even if only for that purpose.

It remains important to make significant progress on the assimilation of satellite data as only a minute fraction of the incoming data is assimilated. Progress is needed on 
the assimilation of radiances in cloudy and rainy conditions (which lead to strong nonlinearities in the observation operator) and also on a better representation of observation error.

Whether it comes as biases or not, model error needs to be taken into account but it is not so obvious to know how to represent and estimate it. The use of multi-model ensembles as provided by the TIGGE database should be explored to estimate the much-needed error statistics.

The problems described above are complex and the academic community could certainly contribute significantly. Due to the complexity of data assimilation with real observations, such activities are greatly facilitated by having close collaborations between the academic community and research departments of operational centres. This is to be encouraged as much as possible. Several centres are poised to invest in large computational infrastructures to test methods directly in a quasi-operational context. Exploring new avenues can be performed in a simpler and more controlled environment from which it is easier to understand what is going on.

Finally, this working group seeks to reach out to the community and researchers are invited to participate to a newly created interest group (daos-ig@ec.gc.ca). Proposing or encouraging specific activities is an approach that needs to be pursued. Whether those are observation campaigns or intercomparison experiments is irrelevant: the end result that is sought is to have several interested research groups to focus on key issues relevant for the THORPEX program. For instance, the intercomparison experiment discussed above in relation with T-PARC will bring together several groups with genuine interest in the issues raised. These would lead to specialized workshops and the results could then be reported to more general meeting like the THORPEX Symposia or data assimilation conferences. The important involvement of the data assimilation research departments of the operational centres provides a good insurance that this knowledge will be transferred to operational systems.

Acknowledgements. We would like to acknowledge A. Doerenbecher, G. N. Petersen, M. Weissman, D. Parsons, P. Drobinski, P. Cocquerez, D. Mansfield and S. Pellerin who provided figures or references for this paper. We would also like to thank N. Fourrié and J. Sutherby for their careful reading of the manuscript.

Edited by: O. Talagrand

Reviewed by: A. Lorenc and another anonymous referee

\section{References}

Atlas, R. A.: Atmospheric observations and experiments to assess their usefulness in data assimilation, J. Meteorol. Soc. Jpn., 75, 111-130, 1997.

Auligné, T., McNally, A. P., and Dee, D. P.: Adaptive bias correction for satellite data in a numerical weather prediction system, Q. J. Roy. Meteorol. Soc., 133, 631-642, 2007.

Baker, N. L. and Daley, R.: Observation and background adjoint sensitivity in the adaptive observation targeting problem, Q. J. Roy. Meteorol. Soc., 126, 1431-1454, 2000.
Bauer, P., Lopez, P., Salmond, D., Benedetti, A., Saarinen, S., and Bonazzola, M.: Implementation of 1D+4D-Var assimilation of precipitation-affected microwave radiances at ECMWF. II: 4DVar, Q. J. Roy. Meteorol. Soc., 132, 2307-2332, 2006.

Bishop, C. H., Etherton, B. J., and Majumdar, S. J.: Adaptive sampling with the Ensemble Transform Kaman Filter. Part I: Theoretical aspects, Mon. Weather Rev., 129, 420-436, 2001.

Bishop, C. H., Reynolds, C. A., and Tippett, M. K.: Optimization of the Fixed Global Obserbing Network in a Simple Model, J. Atmos. Sci., 1471-1489, 2003.

Bormann, N., Saarinen, S., Kelly, G., and Thépaut, J.-N.: The spatial structure of observation error in atmospheric motion vectors from geostationary satellite data, Mon. Weather Rev., 131, 706718, 2003.

Bouttier, F. and Kelly, G.: Observing-system experiments in the ECMWF 4D-Var data assimilation system, Q. J. Roy. Meteorol. Soc., 127,1469-1488, 2001.

Buehner, M.: Ensemble-derived stationary and flow-dependent background error covariances: Evaluation in a quasi-operational NWP setting, Q. J. Roy. Meteorol. Soc., 131, 1013-1044, 2005.

Buizza, R., Cardinali, C., Kelly, G., and Thépaut, J.-N.: The value of observations. Part II: The value of observations located in singular vector-based target areas, Q. J. Roy. Meteorol. Soc., 133 1817-1832, 2007.

Cardinali, C. and Buizza, R.: Forecast skill of targeted observations: a singular-vector-based diagnostics, J. Atmos. Sci., 60, $1927-$ 1940, 2003.

Cardinali, C. and Buizza, R.: Observation sensitivity to the analysis and the forecast: a case study during ATreC targeting campaign, First THORPEX International Science Symposium, 6-10 December 2004, Montreal, Canada, WMO TD 1237 WWRP/THORPEX No. 6, 2004.

Cardinali, C., Pezzuli, S., and Andersson, E.: Influence matrix diagnostic of a data assimilation system, Q. J. Roy. Meteorol. Soc., 130, 2767-2786, 2004.

Cardinali, C. R. Buizza, G. Kelly, M. Shapiro and J-N. Thépaut, 2007: The value of observations. Part III: influence of weather regimes on targeting, Q. J. Roy. Meteorol. Soc., 133, 1833-1842, 2007.

Chapnik, B., Desroziers, G., Rabier, F., and Talagrand, O.: Diagnosis and tuning of observational error statistics in a quasi operational data assimilation setting, Q. J. Roy. Meteorol. Soc., 132, 543-565, 2006.

Davies, H. C.: Large-scale weather systems: A future research priority, Adv. Atmos. Sci., 23, 832-841, 2006.

Dee, D. P., Rukhovets, L., Todling, R., da Silva, A. M., and Larson J. W.: An adaptive buddy check for observational quality control, Q. J. Ror. Meteorol. Soc., 127, 2451-247, 2001.

Dee, D. P.: Bias and data assimilation, Q. J. Roy. Meteorol. Soc., 131, 3323-3343, 2005.

Desroziers, G., Hello, G., and Thépaut, J.-N.: A 4D-Var Reanalysis of FASTEX. Q. J. Roy. Meteorol. Soc., 129, 1301-1315, 2003.

Desroziers, G., Berre, L., Chapnik, B., and Poli, P.: Diagnosis of observation, background and analysis-error statistics in observation space, Q. J. Roy. Meteorol. Soc., 131, 3385-3396, 2005.

Doerenbecher, A. and Bergot, T.: Sensitivity to observations applied to FASTEX cases, Nonlinear Processes in Geophysics, 8(6), 467-481, 2001.

Doerenbecher, A., Leutbecher, M., and Richardson, D. S.: Com- 
parison of observation targeting predictions during the ATReC, Proceedings of the First International THORPEX Science Symposium, 6-10 Dec. 2004, Montreal, Canada, 2004.

Drobinski, P., Parsons, D., Cocquerez, P., Cardonne, A., Cole, H., Fox, J., Hock, T., Lefevre, J.-P., Redelsperger, J.-L., Romberg, K., VanAndel, J., and Venel, S. S.: Des ballons atmosphériques traquent la mousson africaine, La Météorologie, $\mathrm{nr}$ 55, 2-3 Novembre 2006, 2006.

Etherton, B. J. and Bishop, C. H.: Resilience of hybrid ensemble/3DVAR analysis schemes to model error and ensemble covariance error, Mon. Weather Rev., 132, 1065-1080, 2004.

Fisher, M.: Estimation of entropy reduction and degrees of freedom for signal for large variational analysis systems, ECMWF Tech. Memo., 397, 18 pp., 2003.

Fisher, M., Leutbecher, M., and Kelly, G. A.: On the Equivalence between Kalman Smoothing and Weak-Constraint Fourdimensional Variational Data Assimilation, Q. J. Roy. Meteorol. Soc., 131, 3235-3246, 2005.

Fourrié, N. and Rabier, F.: Cloud characteristics and channel selection for IASI radiances in meteorologically sensitive areas, Q. J. Roy. Meteorol. Soc., 130, 1839-1856, 2004.

Fourrié, N., Marchal, D., Rabier, F., Chapnik, B., and Desroziers, G.: Impact study of the 2003 North Atlantic THORPEX Regional Campaign, Q. J. Roy. Meteorol. Soc., 132, 275-295, 2006.

Frehlich, R.: Adaptive data assimilation including the effect of spatial variations in observation error, Q. J. Roy. Meteorol. Soc., 132, 1225-1257, 2006.

Hamill, T. M. and Snyder, C.: Using improved background-error covariances from an ensemble Kalman filter for adaptive observations, Mon. Weather Rev., 130, 1552-1572, 2002.

Houtekamer, P. L. and Mitchell H. L.: Ensemble Kalman Filtering, Q. J. Roy. Meteorol. Soc., 131, 3269-3289, 2005.

Hunt, B. R., Kostelich, E. J., and Szunyogh, I.: Efficient data assimilation for a spatiotemporal chaos: a local ensemble transform Kalman filter, Physica D, 230, 112-126, 2007.

Karbou, F., Gérard, E., and Rabier, F.: Microwave land emissivity and skin temperature for AMSU-A and -B assimilation over land, Q. J. Roy. Meteorol. Soc., 132, 2333-2355, 2006.

Kelly, G., Thépaut, J.-N., Buizza, R., and Cardinali, C.: The value of observations. Part I: the value of observations taken over the oceans, Q. J. Roy. Meteorol. Soc., 133, 1803-1815, 2007.

Kelly, G.: Evaluation of the impact of the space component of the Global Observation System through Observing System Experiments, ECMWF Newsletter Autumn 2007.

Khare, S. P. and Anderson,, J. L.: A methodology for fixed observational network design: theory and application to a simulated global prediction system, Tellus, 58A, 523-537, 2006.

Langland, R. H. and Baker, N. L.: Estimation of observation impact using the NRL atmospheric variational data assimilation adjoint system, Tellus, 56A,189-201, 2004.

Langland, R.: Issues in targeted observing, Q. J. Roy. Meteorol. Soc., 131, 3409-3425, 2005.

Langland, R.: Observation impact during the North Atlantic TreC2003, Mon. Weather Rev., 133, 2297-2309, 2005.

Leutbecher, M.: A Reduced Rank Estimate of Forecast Error Variance Changes due to Intermittent Modifications to the Observing Network, J. Atmos. Sci., 729-742, 2003.

Liu, Z.-Q. and Rabier, F.: The potential of high-density observations for numerical weather prediction: A study with simulated observations, Q. J. Roy. Meteorol. Soc., 129, 3013-3035, 2003.

Lorenc, A. C.: The potential of the ensemble Kalman filter for NWP- a comparison with 4D-Var, Q. J. Roy. Meteorol. Soc., 129, 383-3202, 2003.

Majumdar, S. J., Bishop, C. H., Buizza, R., and Gelaro, R.: A comparison of ensemble-transform Kalman-filter targeting guidance with ECMWF and NRL total energy singular vector guidance, Q. J. Roy. Meteorol. Soc., 128, 2527-2549, 2002.

Majumdar, S. J., Aberson, S. D., Bishop, C. H., Buizza, R., Peng, M. S., and Reynolds, C. A.: A Comparison of Adaptive Observing Guidance for Atlantic Tropical Cyclones, Mon. Weather Rev., 134, 2354-2372, 2006.

Mansfield, D., Richardson, D., and Truscott, B.: An overview of the Atlantic THORPEX Regional Campaign, ATReC. In Proceedings of the THORPEX Science Symposium, 6 to 10 December 2004, Montreal, Canada, World Meteorological Organization, Geneva, Switzerland, 2005.

Masutani, M., Andersson, E., Terry, J., Reale, O., Jusem, J. C., Riishojgaard, L. P., Schlatter, T., Stoffelen, A., Woollen, J., Lord, L., Toth, Z., Son, Y., Kleist, D., Xie, Y., Prive, N., Liu, E., Sun, H., Emmitt, D., Greco, S., Wood, S. A., Marseille, G. J., Errico, R., Yang, R., McConaughy, G., Devenyi, D., Weygandt, S., Anantharaj, V., Hill,C., Fitzpatrick, P., Weng, F., Zhu, T., and Boukabara, S.: Joint Observing System Simulation Experiments, Proc. "18th Conf. on Numerical Weather Prediction", 25-29 June 2007, Park City, Utah, Available from the American Meteorological Society, 2007.

Ochotta, T., Gerhardt, C., Saupe, D., and Wergen, W.: Adaptive thinning of atmospheric observations in data assimilation with vector quantization and filtering methods, Q. J. Roy. Meteorol. Soc., 131, 3427-3437, 2005.

Petersen, G. N. and Thorpe, A. J.: The impact on weather forecasts of targeted observations during A-TreC, Q. J. Roy. Meteorol. Soc., 133, 417-431, 2007.

Purser, R. J. and Huang, H. L.: Estimating effective data density in a satellite retrieval or an objective analysis, J. Appl. Meteorol., 32, 1092-1107, 1993.

Rabier, F., Fourrié, N., Chafai, D., and Prunet P.: Channels selection methods for infrared atmospheric sounding interferometer radiances, Q. J. Roy. Meteorol. Soc., 128, 1011-1027, 2002.

Rodgers, C. D.: Inverse methods for Atmospheres: Theories and practice, World Scientific Publ., Singapore, 238 pp., 2000.

Szunyogh, I., Toth, Z., Morss, R., Majumdar, S., Etherton, B. J., and Bishop, C. H.: The effect of targeted dropsonde observation during the 1999 Winter Storm Reconnaissance Program, Mon. Weather Rev., 128, 3520-3537, 2000.

Tan, D.G. H., Andersson, E., Fisher, M., and Isaksen, L.: Observing system impact assessment using a data assimilation ensemble technique: Application to the ADM-Aeolus wind profiling mission, Q. J. Roy. Meteorol. Soc., 133, 381-390, 2007.

Tsyrulnikov, M. D.: Stochastic modelling of model errors: a simulation study, Q. J. Roy. Meteorol. Soc. , 131, 3345-3371, 2005.

Weissmann, M. and Cardinali, C.: Impact of airborne Doppler lidar observations on ECMWF forecasts, Q. J. Roy. Meteorol. Soc., 133, 107-116, 2007.

Zhu, Y. and Gelaro, R.: Observation sensitivity calculations using the adjoint of the grid-point statistical interpolation (GSI) analysis system, Mon. Weather Rev., 136, 335-351, 2008. 